\title{
Editorial
}

\section{The Diversity of Australasian Rehabilitation Research}

I am pleased to welcome readers to this issue of the journal. The contributions herein reflect the intellectual rigour and scholarly debate that characterises rehabilitation research in this part of the world. Academic research is often criticised for being 'blue sky' research that lacks relevance for industry and for practitioners working at the coal face. The contributions in this issue, however, are compelling examples of research that effectively spans that divide and links the scientist and the practitioner.

The first two articles address issues of 'safety' in occupational drivers, and in construction work settings. The first article by Andrew Wills and colleagues considers the structure and applicability of a pre-existing safety climate assessment tool modified for drivers and the authors conclude that their results 'have a direct and immediate usefulness to rehabilitation and disability management as a planning measure and proactive early intervention strategy'. The related article by Bert Biggs and his colleagues examines construction site safety culture via a qualitative study with a number of focus groups and they rightly conclude that their data provide '... a much needed understanding of the dynamics of close quarter and dangerous working environments which routinely injure many thousands annually'. The third article by Lynnette Guy and her colleagues concerns the pressing problem of successful rehabilitation following whiplash injuries. As they point out, the financial costs of whiplash injuries are extensive, and the recovery process is 'uncertain and controversial'. The mediating effects of pain self efficacy on the relationship between neck disability and difficulty in return to work, highlight the powerful influence of the self efficacy construct. This clearly has implications for intervention at various levels where efficacy expectations, outcome expectations, and outcome values can be identified and modified in appropriate ways. The fourth contribution by Tim Coombs and Paul Hirini, 'Training in the Health of the Nation Outcome Scales ...' describes some of the issues around the training of mental health professionals in the use of an assessment tool, the Health of the Nations Outcome Scales. As the authors note, the article serves to emphasise the importance of '...continued consideration of the role that cultural context plays in the lives and presentation of mental health consumers'. The remaining two contributions are a discussion between researchers in response to an article published in this journal titled 'The assessment of pain beliefs and their role in predicting recovery from repetitive strain injury', Volume 10, Number 1 (2004). Greg Murphy raises a number of issues about the design of the study and the conclusions that were drawn to which Lucinda Allison responds. The final article is a comprehensive review of PTSD related issues among veterans.

In summary I am pleased to be associated with another issue of this journal given the quality and diversity of research represented here. I would encourage readers to make submissions to the journal. If readers or groups of readers have ideas for special issues of the journal, that is, issues that might concentrate on a particular theme or context, then I would also encourage them to get in contact with me and I would be happy to facilitate the production of such an issue.

Ross Flett PhD, Editor 
